OPEN ACCESS

Edited by:

Mingzhu Yin,

Central South University, China

Reviewed by:

José Medina-Echeverz,

Bavarian Nordic, Germany

Ali Bettaieb,

Université de Sciences Lettres de

Paris, France

${ }^{*}$ Correspondence:

Shengjun Wang

sjwjs@ujs.edu.cn

Specialty section: This article was submitted to

Cancer Immunity and Immunotherapy,

a section of the journal

Frontiers in Immunology

Received: 02 April 2021

Accepted: 17 May 2021

Published: 02 June 2021

Citation:

Cui Y, Cai J, Wang W and Wang $S$ (2021) Regulatory Effects of Histone

Deacetylase Inhibitors on Myeloid-

Derived Suppressor Cells.

Front. Immunol. 12:690207.

doi: 10.3389/fimmu.2021.690207

\section{Regulatory Effects of Histone Deacetylase Inhibitors on Myeloid- Derived Suppressor Cells}

\author{
Yudan Cui ${ }^{1,2}$, Jingshan Cai ${ }^{1,2}$, Wenxin Wang $^{2}$ and Shengjun Wang ${ }^{1,2 *}$ \\ ${ }^{1}$ Department of Laboratory Medicine, The Affiliated People's Hospital, Jiangsu University, Zhenjiang, China, ${ }^{2}$ Department of \\ Immunology, Jiangsu Key Laboratory of Laboratory Medicine, School of Medicine, Jiangsu University, Zhenjiang, China
}

Histone deacetylase inhibitors (HDACls) are antitumor drugs that are being developed for use in clinical settings. HDACls enhance histone or nonhistone acetylation and promote gene transcription via epigenetic regulation. Importantly, these drugs have cytotoxic or cytostatic properties and can directly inhibit tumor cells. However, how HDACls regulate immunocytes in the tumor microenvironment, such as myeloid-derived suppressor cells (MDSCs), has yet to be elucidated. In this review, we summarize the effects of different HDACls on the immunosuppressive function and expansion of MDSCs based on the findings of relevant studies.

Keywords: acetylation, histone deacetylases, inhibitors, myeloid-derived suppressor cells, immunosuppression

\section{INTRODUCTION}

Myeloid-derived suppressor cells (MDSCs) are heterogeneous cells derived from bone marrow that can suppress the immune response $(1,2)$. MDSCs are produced in large quantities under pathological conditions, such as inflammation and cancer. The accumulation of MDSCs is a complex and gradual phenomenon that is regulated by many factors (3). MDSCs are composed of two major types of cells: the granulocytic or polymorphonuclear type (PMN-MDSCs), which are similar to neutrophils in phenotype and morphology, and the monocytic type (M-MDSCs), which are similar to monocytes in phenotype and morphology. In most types of cancer, PMN-MDSCs account for more than $80 \%$ of all MDSCs, while M-MDSCs are direct promoters of tumor metastasis (4). In mice, MDSCs are more common in tumors of the bone marrow, spleen, liver and other organs. PMN-MDSCs and M-MDSCs are defined as $\mathrm{CD} 11 \mathrm{~b}^{+} \mathrm{Ly} 6 \mathrm{G}^{+} \mathrm{Ly} 6 \mathrm{C}^{\mathrm{lo}}$ and

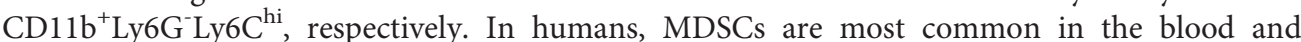
tumors of various organs. In peripheral blood mononuclear cells, PMN-MDSCs are defined as $\mathrm{CD} 11 \mathrm{~b}^{+} \mathrm{CD} 14^{-} \mathrm{CD} 15^{+}$or $\mathrm{CD} 11 \mathrm{~b}^{+} \mathrm{CD} 14^{-} \mathrm{CD} 66 \mathrm{~b}^{+}$, while M-MDSCs are defined as


containing more immature progenitor cells that have been proposed to be defined by 'early-stage MDSCs' (e-MDSCs). However, the same type of cells have yet to be identified in mice (5). The signals driving the development of MDSCs occur in two partially overlapping stages (6). In the first stage, the expansion and regulation of bone marrow cells occur in the bone marrow and spleen, 
while the second stage is characterized by the transformation of neutrophils and monocytes into pathologically activated MDSCs, which primarily occurs in peripheral tissues (7). Several factors participate in MDSC-mediated immunosuppression, including arginase-1 (Arg-1), inducible nitric oxide synthase 2 (iNOS), transforming growth factor $\beta$ (TGF- $\beta$ ), interleukin-10 (IL-10), cyclooxygenase 2 (COX2) and indoleamine 2,3-dioxygenase (IDO) $(8,9)$. Although MDSCs are involved in the suppression of different cells in the immune system, $\mathrm{T}$ cells are the primary targets of MDSCs. Both PMN-MDSCs and M-MDSCs can reduce the production of L-arginine through the expression of Arg-1 and iNOS, thereby inhibiting the function of T cells (10, 11). Furthermore, M-MDSCs and PMN-MDSCs also take advantage of different immunosuppressive mechanisms. MMDSCs use NO and produce related cytokines to inhibit the ability of T cells to eliminate antigens (12), while PMN-MDSCs primarily inhibit the immune response in an antigen-specific manner. The induction of antigen-specific T cell tolerance is one of the primary characteristics of PMN-MDSCs $(13,14)$, and reactive oxygen species (ROS) production is crucial for this activity (8). In recent years, an understanding of the clinical importance of MDSCs has emerged. An initial study monitored MDSCs from cancer patients and analyzed the total MDSC population. The results showed that the number of peripheral blood MDSCs was positively correlated with the tumor stage and tumor burden of colorectal, breast, thyroid and nonsmall cell lung cancers $(3,15-21)$. In melanoma and hepatocellular carcinoma, both PMN-MDSCs and M-MDSCs were shown to be associated with a poorer prognosis $(3,22,23)$. In nonsolid tumors, M-MDSC numbers were associated with reduced survival in multiple myeloma, Hodgkin's lymphoma, nonHodgkin's lymphoma, and diffuse large B-cell lymphoma (2426). Therefore, therapeutics targeting MDSCs have become an important means of tumor immunotherapy by inhibiting their differentiation, expansion and activity.

Immune checkpoint inhibitors, such as anti-PD-1 and anti-CTLA-4, have shown success in eradicating cancer by enhancing immune activation, but primary and secondary resistance are still problems (27). Epigenetic treatments for cancer include histone deacetylase inhibitors (HDACIs), DNA methyltransferase inhibitors (DNMTIs) and histone methyltransferase inhibitors (HMTIs), which can stimulate tumor cells and improve the antitumor response by host immune cells. Epigenetic treatments can improve the response of tumor patients to immune checkpoint blockade therapy (28). DNMTIs have been reported to be effective in the treatment of hematological malignancies in clinical studies (29), while HMTIs have been shown to play a role in the treatment of multiple myeloma (30). However, some DMNTIs and HMTIs have not shown clinical efficacy. HDACIs are a different class of small molecule drugs that can have a wide range of effects on tumor cells, including cell cycle arrest, apoptosis, cell differentiation, autophagy and antiangiogenesis (31). HDACIs can inhibit HDACs, and because these drugs have a more pronounced effect on the proliferation of malignant cells than nonmalignant cells, there is increasing interest in developing these drugs, especially as antitumor treatments. In recent studies, many researchers have found that HDACIs also have significant effects on host immunosuppressive cells. As MDSCs are important immunosuppressive cells in the tumor microenvironment (32), it is worth investigating the regulatory effects of HDACIs these cells.

\section{ACETYLATION}

Lysine acetylation is an evolutionarily conserved posttranslational modification that occurs in prokaryotes and eukaryotes. In general, two different types of protein acetylation occur in cells. In humans, $80-90 \%$ of proteins undergo cotranslational acetylation at the $\mathrm{N} \alpha$ end of the nascent polypeptide chain (43-45). The other common type of protein acetylation occurs at the $\epsilon$-amino group of lysine. Acetylation was first discovered in histones (46). Subsequently, researchers observed acetylation modifications on nonhistones and identified histone acetyltransferases (HATs) and histone deacetylases (HDACs). In the past decade, advances in proteomics based on mass spectrometry have greatly expanded the classification of endogenous acetylated proteins, provided an objective perspective for the study of acetylation, and provided new insights into the scope and regulation of nonhistone acetylation. To reflect the degree of nonhistone acetylation, HATs and HDACs were renamed lysine acetyltransferases (KATs) and lysine deacetylases (KDACs), respectively (47) (ordinarily, the terms HATs and HDACs are used). Acetylation is a dynamic and reversible process involving both KATs and KDACs. KATs are responsible for covalently attaching an acetyl group to the lysine residue of a protein and are figuratively called "writers", while KDACs mediate the removal of this acetyl group and are called "erasers". Acetylation is the addition of acetyl groups to lysine residues in a protein that occurs in the presence of acetyl transferase. Acetylation is an important type of posttranslational modification for acetyl-CoA metabolism and cell signal transduction. In addition, acetylation is a widespread regulatory mechanism mediated by posttranslational modification in the subcellular organelles of the nucleus or cytoplasm and is involved in many processes, such as transcription, chemotaxis, metabolism, cell signal transduction, stress response, proteolysis, cell apoptosis, and neuron development (47). Evidence has shown that acetylation is one of the most important modifications used to alter protein activity and precisely regulate and control cellular functions.

\section{HISTONE DEACETYLASES}

HDACs can mediate the deacetylation of histones and nonhistone proteins and are a class of proteases that play important roles in chromosome structural modifications and gene expression regulation (48). Under normal conditions, the acetylation of histones is beneficial for the dissociation of DNA 
and dense histone octamers, allowing the nucleosome structure to relax so that various transcription factors and cooperative transcription factors can bind to specific DNA binding sites and activate gene transcription. The deacetylation of histones has the opposite effect (49). In addition to regulating histone modification, HDACs also regulate the posttranslational acetylation of many nonhistones, including transcription factors, chaperones, and signaling molecules, leading to changes in protein stability, protein-protein interactions, and protein-DNA interactions (50). There are four classes of HDACs. Class I includes HDAC1, HDAC2, HDAC3 and HDAC8. Class II is further divided into IIa and IIb, with HDAC4, HDAC5, HDAC7 and HDAC9 belonging to class IIa, while class IIb includes HDAC6 and HDAC10. Class III is composed of sirtuin1-7, and class IV includes HDAC11 only. Classes I, II and IV enzymes are zinc ion dependent, while class III members are zinc ion independent (51). It is worth noting that nearly half of all deacetylases have weak or no deacetylase activity or target other types of acylation (47).

\section{HISTONE DEACETYLASE INHIBITORS}

HDACIs can inhibit the deacetylation of histones or nonhistone proteins and have direct inhibitory effects on tumor cells. Inhibiting HDACs can regulate the balance between proapoptotic and antiapoptotic proteins, leading to the death of tumor cells (52). While HDACIs have direct inhibitory effects on tumor cells, they can also regulate various components of the host immune system (53). Some researchers have found that the treatment of cancer patients with HDACIs can reduce the number of lymphocytes, indicating that HDACIs are immunocytotoxic $(54,55)$. On the other hand, some researchers have shown that HDACIs promote immune activity and can enhance cancer immunotherapy (56-58). Theoretically, targeted inhibition of HDACs is closely associated with adverse outcomes after trauma and can optimize treatment outcomes while reducing complications (59). Many isotype-specific HDACIs are now available and are undergoing clinical trials as antitumor agents (60). HDACIs can be structurally classified into at least four categories (hydrochlorates, cyclic peptides, fatty acids, and benzoamides) and can also be classified according to their HDAC specificity. Broad-spectrum HDACIs include panobinostat, belinostat, resminostat and trichostatin A. Butyrate and valproate inhibit class I and IIa HDACs. Romidepsin, entinostat (ENT) and mocetinostat are considered class I specific, and tubacin is HDAC6 specific (51). Due to the zinc ion-dependent nature of the domains of class I, II and IV HDACs, inhibitors occupying the zinc ionbinding site of the catalytic center will inhibit the activity of these enzymes. These HDACIs contain a pharmacophore, a cap structure, a linking unit and a zinc ion-binding group to chelate cations in the catalytic region of the target HDACs (27). Trichostatin A, vorinostat, belinostat, dacinostat, panobinostat and givinostat are HDACIs. Recent studies have shown that HDACIs also have crucial effects on host immunosuppressive cells, with MDSCs being important immunosuppressive cells in the tumor microenvironment (Table 1).

\section{EFFECTS OF HDACIs ON MDSCs}

\section{Entinostat}

ENT is a specific inhibitor of class I HDACs that targets immunosuppressive cells in the tumor microenvironment (61). ENT has been reported to have immunoregulatory activity (62) and has been used in the clinical treatment of breast and nonsmall cell lung cancers $(63,64)$. The clinical drug development of ENT focuses on the resistance mechanism of breast cancer to endocrine therapy and HER2-targeted drugs (63). Importantly, ENT can inhibit tumor cell proliferation, which can induce mitochondrial damage and lead to apoptosis. ENT increases the sensitivity of lung cancer cells to tumor necrosis factor-related apoptosis-inducing ligands and downregulates the expression of the antiapoptotic genes $\mathrm{Bcl}-2$ and XIAP (64).

Using lung and renal cell carcinoma models, Orillion A and colleagues observed that the total number of MDSCs in tumors increased in the presence of ENT alone but only slightly increased after treatment with ENT combined with anti-PD-1. In addition, there was also a decline in immunosuppressive functions, showing that ENT can inhibit the levels of Arg-1, iNOS and COX2, thereby reducing the immunosuppressive

TABLE 1 | Effects of HDACls on MDSCs.

\begin{tabular}{|c|c|c|c|}
\hline HDACI & Classification & Effects on MDSCs & Ref \\
\hline & & & (34) \\
\hline & & M-MDSC migration inhibited & $(36)$ \\
\hline Mocetinostat & Class I/IV & total number of MDSCs decreased & (37) \\
\hline Vorinostat & Class $\mathrm{I} / \mathrm{II}$ & $\begin{array}{l}\text { MDSC apoptosis (at higher vorinostat concentrations) } \\
\text { total number of MDSCs amplified (at lower vorinostat concentrations) }\end{array}$ & $\begin{array}{l}(38) \\
(39)\end{array}$ \\
\hline \multirow[t]{2}{*}{ Trichostatin A } & $\begin{array}{l}\text { Broad spectrum } \\
\text { (except HDAC8) }\end{array}$ & total number of MDSCs amplified (0.1-10 nM TSA) & (39) \\
\hline & & PMN-MDSC number decreased & (42) \\
\hline
\end{tabular}


effects of MDSCs (62). After treatment with ENT, the tumor-free survival of HER/neu transgenic breast cancer and Panco2 metastatic pancreatic cancer mouse models was significantly improved. ENT combined treatment with anti-PD-1 and antiCTLA-4 was shown to inhibit the VEGF, ErbB and mTOR pathways in PMN-MDSCs as well as the activity of STAT3 and the activity of Arg-1 (33). Tomita et al. reported that the number of circulating PMN-MDSCs and M-MDSCs decreased in samples from breast cancer patients treated with ENT combined with an aromatase inhibitor (65). However, the immunosuppressive activity of PMN-MDSCs could specifically be reduced by ENT treatment, and there was no effect on MMDSCs (66). The microenvironment before tumor metastasis has been shown to be established through the activities of MMDSCs, suggesting that the number of M-MDSCs and nichepromoting molecules in the lung tissue before tumor metastasis can be reduced by low-dose 5-azacytidine (100 nM) and lowdose ENT $(50 \mathrm{nM})$ treatment. Interestingly, the gene set related to the chemokine axis and immune cell migration was observed to be significantly altered by low-dose ENT treatment, and the expression of CCR2 in M-MDSCs in the bone marrow and lung was significantly downregulated after low-dose ENT treatment (34). CCR2 is a key regulator of the migration of M-MDSCs from the bone marrow to the tumor environment, suggesting that the transport of M-MDSCs to the premetastatic lung may be affected by low-dose ENT therapy at least partially through the downregulation of CCR2 $(67,68)$. In short, ENT can inhibit the function of PMN-MDSCs and the metastasis of MMDSCs (Figure 1).

\section{Valproic Acid}

Valproic acid (VPA) is an anticonvulsant drug (69) and an HDACI $(70,71)$ that targets HDAC class I enzymes (HDAC1,
2 and 3). In vitro experiments by Xie $\mathrm{Z}$ et al. showed that VPA treatment can reduce the proportion of PMN-MDSCs, inhibit the immunosuppressive function of MDSCs in a dose-dependent manner and also reduce the level of Arg-1 by inhibiting IL- 4 R $\alpha$ expression, thereby weakening the immunosuppressive function of MDSCs (35). In a recent study, VPA was shown to downregulate CCR2 expression in M-MDSCs, and the tumor invasion ability of these cells was also reduced (36). In addition, VPA was shown to decrease the immunosuppressive effects of MDSCs on $\mathrm{CD}^{+} \mathrm{T}$ cells and NK cells, and the ability of these cells to kill tumors was also enhanced (36). Moreover, treatment with VPA combined with an anti-PD-L1 antibody blocked the immunosuppressive functions of MDSCs by activating IRF1/ IRF8 (72).

\section{Mocetinostat}

Mocetinostat is a selective inhibitor of class I/IV HDAC, proteins involved in the epigenetic silencing of immunoregulatory genes in tumors and immune cells. The target gene promoters of mocetinostat are occupied by class I HDACs, and an increase in active histone markers is observed after mocetinostat treatment (37). Briere D and colleagues suggested that the number of MDSCs and Tregs could be reduced by mocetinostat treatment, with an increase in $\mathrm{CD}^{+} \mathrm{T}$ cells observe in a tumor-bearing mouse model of colorectal cancer (37). However, the exact mechanism of action of mocetinostat remains unclear.

\section{Vorinostat}

Vorinostat (SAHA) is a nonspecific inhibitor of class I and class II HDACs and was the first HDACI drug approved by the Food And Drug Administration for clinical use in patients with cutaneous $\mathrm{T}$ cell lymphoma (73). Vorinostat can also reduce
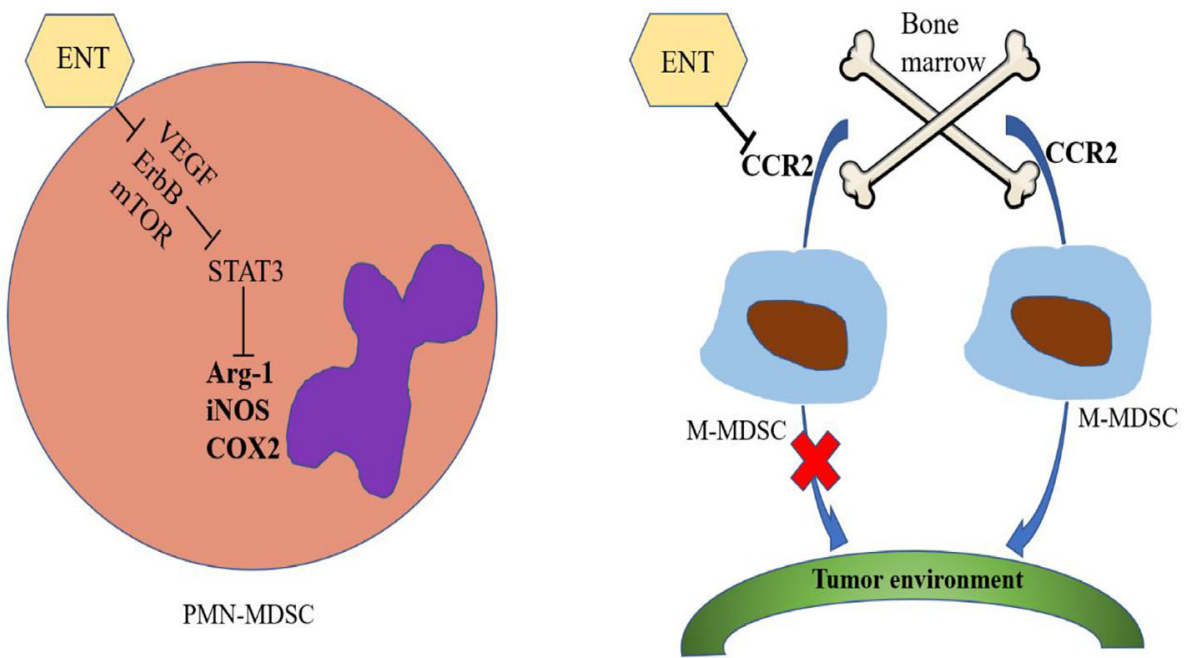

FIGURE 1 | Effects of entinostat on MDSCs. Entinostat inhibits the VEGF, ErbB and mTOR pathways in PMN-MDSCs, thereby inhibiting the activity of STAT3, which in turn reduces the activities of Arg-1, iNOS and COX2. Entinostat therapy inhibits the transport of M-MDSCs from bone marrow to the tumor environment by downregulating CCR2 expression. 
acute graft-versus-host disease (GVHD) after allogeneic bone marrow transplantation by inhibiting the production of proinflammatory cytokines such as TNF- $\alpha$, IL- 1 and IFN- $\gamma(73)$.

MDSCs were shown to be induced by both GM-CSF and vorinostat-induced tumor pressure in vitro, which can mediate MDSC apoptosis and contradicts the results of other researchers, possibly because different concentrations of vorinostat were used (38). In a spontaneous transgenic mouse melanoma model, treatment with vorinostat resulted in a significant delay in disease onset, downregulation of chemokine (c-c motif) ligand 2 (CCL2) and the recruitment of MDSCs (74). Kroesen M and colleagues showed that the number of M-MDSCs in the tumor microenvironment of $9464 \mathrm{D}$ tumor-bearing mice could be reduced by vorinostat treatment. Thus, vorinostat can create a permissible tumor microenvironment for tumor-directed $\mathrm{mAb}$ therapy by increasing the number of macrophage effector cells expressing high levels of Fc receptors (75).

\section{CG-745}

CG-745 is a specific inhibitor of class I and class IIb HDACs that exhibits anticancer effects on pancreatic, colorectal and nonsmall cell lung cancers (56). Kim YD and colleagues analyzed the distribution of immune cells in the tumor microenvironment and spleen and reported that CG-745 could inhibit M2 macrophage polarization and reduce the number of MDSCs (40). Therefore, the cytotoxicity of PBMCs and IFN- $\gamma$ expression in Jurkat T cells could be increased by CG-745. H3 acetylation, which is an important factor during the differentiation of naïve $\mathrm{CD}^{+} \mathrm{T}$ cells into memory $\mathrm{T}$ cells, was also induced (76).

\section{ACY241}

ACY241 is a specific inhibitor of HDAC6 that inhibits multiple myeloma when used in combination with immunoregulatory drugs and proteasome inhibitors (41). After treatment with ACY241, the number of MDSCs in patients with multiple myeloma was shown to significantly decrease. Bcl6 expression in $\mathrm{CD}^{+} \mathrm{T}$ cells may be enhanced by ACY 241 through activation of the AKT/mTOR/NF- $\mathrm{KB}$ signaling pathway in $\mathrm{CD}^{+} \mathrm{T}$ cells, thereby enhancing $\mathrm{CD}^{+} \mathrm{T}$ cell activity (41).

\section{Trichostatin A}

Trichostatin A (TSA) is a natural antifungal metabolite produced by Streptomyces and is a broad-spectrum HDACI with no effect on HDAC8 (77). Rosboroug BR et al. observed that after GMCSF-induced mouse bone marrow cells were treated with TSA (0.1-10 nM) and vorinostat (10-500 nM), CD11b+ GR1+ cells and MDSCs were strongly amplified (39). After TSA treatment of experimental autoimmune encephalomyelitis, PMN-MDSCs were present in reduced numbers in secondary lymphoid

\section{REFERENCES}

1. Liu G, Bi Y, Shen B, Yang H, Zhang Y, Wang X, et al. SIRT1 Limits the Function and Fate of Myeloid-Derived Suppressor Cells in Tumors by Orchestrating HIF-1alpha-dependent Glycolysis. Cancer Res (2014) 74 (3):727-37. doi: 10.1158/0008-5472.CAN-13-2584 organs and migrated into the spinal cord without affecting monocytes, while the disease symptoms improved (42). Additionally, the numbers of Tregs and MDSCs were reduced in Her2/CT26 tumor-bearing mice treated with TSA (78).

\section{CONCLUSION}

In general, most HDACIs inhibit class I or class II HDACs. Among these molecules, the regulatory effects of entinostat on MDSCs have been reported the most often, probably because entinostat has been put into clinical use. In summary, entinostat inhibits the VEGF, ErbB and mTOR pathways in PMN-MDSCs, thereby inhibiting the activity of STAT3, which in turn reduces the activity of Arg-1, iNOS and COX2. Entinostat also inhibits the transport of M-MDSCs from bone marrow to the tumor environment by downregulating CCR2. Other HDACIs have antitumor effects by reducing the number of MDSCs, but the specific mechanism of action varies. Interestingly, increased concentrations of vorinostat can amplify the number of MDSCs. Why different concentrations of vorinostat lead to different results is worth further research. At the same time, these results suggest that different doses of HDACIs may have different effects, demonstrating that studies of HDACIs must involve strict control of the drug dose. HDACIs have been shown to be effective antitumor agents in clinical studies, but their success has been limited. In addition, these inhibitors can produce side effects, such as platelet reduction, nausea, vomiting, anorexia and fatigue.

In recent years, research on MDSCs has gradually increased, and some researchers regard MDSCs as targets of tumor therapy. Therefore, it is necessary to explore the regulatory effects of HDACIs on MDSCs, which may improve their therapeutic effects toward tumors.

\section{AUTHOR CONTRIBUTIONS}

YC drafted the manuscript. JC and WW discussed and revised the manuscript. SW designed the study and revised the manuscript. All authors contributed to the article and approved the submitted version.

\section{FUNDING}

This work was supported by the Research Project of the Jiangsu Commission of Health (grant No. K2019019) and the Jiangsu Province's Key Medical Talents Program (grant No. ZDRCB2016018).

2. Mantovani A. The Growing Diversity and Spectrum of Action of MyeloidDerived Suppressor Cells. Eur J Immunol (2010) 40(12):3317-20. doi: 10.1002/eji.201041170

3. Veglia F, Perego M, Gabrilovich D. Myeloid-Derived Suppressor Cells Coming of Age. Nat Immunol (2018) 19(2):108-19. doi: 10.1038/s41590017-0022-x 
4. Mantovani A, Marchesi F, Malesci A, Laghi L, Allavena P. TumourAssociated Macrophages as Treatment Targets in Oncology. Nat Rev Clin Oncol (2017) 14(7):399-416. doi: 10.1038/nrclinonc.2016.217

5. Bronte V, Brandau S, Chen S, Colombo M, Frey A, Greten T, et al. Recommendations for Myeloid-Derived Suppressor Cell Nomenclature and Characterization Standards. Nat Commun (2016) 7:12150. doi: 10.1038/ ncomms 12150

6. Condamine T, Mastio J, Gabrilovich DI. Transcriptional Regulation of Myeloid-Derived Suppressor Cells. J Leukoc Biol (2015) 98(6):913-22. doi: 10.1189/jlb.4RI0515-204R

7. Veglia F, Sanseviero E, Gabrilovich DI. Myeloid-Derived Suppressor Cells in the Era of Increasing Myeloid Cell Diversity. Nat Rev Immunol (2021) 1:1-14. doi: 10.1038/s41577-020-00490-y

8. Gabrilovich DI. Myeloid-Derived Suppressor Cells. Cancer Immunol Res (2017) 5(1):3-8. doi: 10.1158/2326-6066.CIR-16-0297

9. Feng S, Cheng X, Zhang L, Lu X, Chaudhary S, Teng R, et al. Myeloid-Derived Suppressor Cells Inhibit T Cell Activation Through Nitrating LCK in Mouse Cancers. Proc Natl Acad Sci USA (2018) 115(40):10094-9. doi: 10.1073/ pnas. 1800695115

10. Bronte V, Serafini P, Mazzoni A, Segal DM, Zanovello P. L-Arginine Metabolism in Myeloid Cells Controls T-Lymphocyte Functions. Trends Immunol (2003) 24(6):301-5. doi: 10.1016/s1471-4906(03)00132-7

11. Kamran N, Kadiyala P, Saxena M, Candolfi M, Li Y, Moreno-Ayala MA, et al. Immunosuppressive Myeloid Cells' Blockade in the Glioma Microenvironment Enhances the Efficacy of Immune-Stimulatory Gene Therapy. Mol Ther (2017) 25(1):232-48. doi: 10.1016/j.ymthe.2016.10.003

12. Gabrilovich DI, Ostrand-Rosenberg S, Bronte V. Coordinated Regulation of Myeloid Cells by Tumours. Nat Rev Immunol (2012) 12(4):253-68. doi: $10.1038 /$ nri3175

13. Koehn B, Apostolova P, Haverkamp J, Miller J, McCullar V, Tolar J, et al. GVHD-Associated, Inflammasome-Mediated Loss of Function in Adoptively Transferred Myeloid-Derived Suppressor Cells. Blood (2015) 126(13):1621-8. doi: 10.1182/blood-2015-03-634691

14. Marigo I, Bosio E, Solito S, Mesa C, Fernandez A, Dolcetti L, et al. TumorInduced Tolerance and Immune Suppression Depend on the C/EBPbeta Transcription Factor. Immunity (2010) 32(6):790-802. doi: 10.1016/ j.immuni.2010.05.010

15. Sun $\mathrm{H}$, Zhou X, Xue $\mathrm{Y}$, Wang K, Shen $\mathrm{Y}$, Mao J, et al. Increased Frequency and Clinical Significance of Myeloid-Derived Suppressor Cells in Human Colorectal Carcinoma. World J Gastroenterol (2012) 18(25):3303-9. doi: 10.3748/wjg.v18.i25.3303

16. Zhang B, Wang Z, Wu L, Zhang M, Li W, Ding J, et al. Circulating and Tumor-Infiltrating Myeloid-Derived Suppressor Cells in Patients With Colorectal Carcinoma. PloS One (2013) 8(2):e57114. doi: 10.1371/ journal.pone.0057114

17. Wang Y, Yin K, Tian J, Xia X, Ma J, Tang X, et al. Granulocytic MyeloidDerived Suppressor Cells Promote the Stemness of Colorectal Cancer Cells Through Exosomal S100A9. Adv Sci (2019) 6(18):1901278. doi: 10.1002/ advs.201901278

18. Diaz-Montero C, Salem M, Nishimura M, Garrett-Mayer E, Cole D, Montero AJ. Increased Circulating Myeloid-Derived Suppressor Cells Correlate With Clinical Cancer Stage, Metastatic Tumor Burden, and DoxorubicinCyclophosphamide Chemotherapy. Cancer Immunol Immunother (2009) 58 (1):49-59. doi: 10.1007/s00262-008-0523-4

19. Angell T, Lechner M, Smith A, Martin S, Groshen S, Maceri D, et al. Circulating Myeloid-Derived Suppressor Cells Predict Differentiated Thyroid Cancer Diagnosis and Extent. Thyroid (2016) 26(3):381-9. doi: $10.1089 /$ thy.2015.0289

20. Tian X, Ma J, Wang T, Tian J, Zhang Y, Mao L, et al. Long Non-Coding RNA HOXA Transcript Antisense RNA Myeloid-Specific 1-HOXA1 Axis Downregulates the Immunosuppressive Activity of Myeloid-Derived Suppressor Cells in Lung Cancer. Front Immunol (2018) 9:473. doi: 10.3389/fimmu.2018.00473

21. Huang A, Zhang B, Wang B, Zhang F, Fan K, Guo Y. Increased CD14(+)HLADR (-/Low) Myeloid-Derived Suppressor Cells Correlate With Extrathoracic Metastasis and Poor Response to Chemotherapy in Non-Small Cell Lung Cancer Patients. Cancer Immunol Immunother (2013) 62(9):1439-51. doi: 10.1007/s00262-013-1450-6
22. Jordan K, Amaria R, Ramirez O, Callihan E, Gao D, Borakove M, et al. Myeloid-Derived Suppressor Cells are Associated With Disease Progression and Decreased Overall Survival in Advanced-Stage Melanoma Patients. Cancer Immunol Immunother (2013) 62(11):1711-22. doi: 10.1007/s00262013-1475-X

23. Li X, Xing Y, Lei A, Xiao Q, Lin Z, Hong Y, et al. Neutrophil Count is Associated With Myeloid Derived Suppressor Cell Level and Presents Prognostic Value of for Hepatocellular Carcinoma Patients. Oncotarget (2017) 8(15):24380-8. doi: 10.18632/oncotarget.15456

24. Wang Z, Zhang L, Wang H, Xiong S, Li Y, Tao Q, et al. Tumor-Induced CD14+ HLA-DR (-/Low) Myeloid-Derived Suppressor Cells Correlate With Tumor Progression and Outcome of Therapy in Multiple Myeloma Patients. Cancer Immunol Immunother (2015) 64(3):389-99. doi: 10.1007/s00262-014-1646-4

25. Wu C, Wu X, Zhang X, Chai Y, Guo Q, Li L, et al. Prognostic Significance of Peripheral Monocytic Myeloid-Derived Suppressor Cells and Monocytes in Patients Newly Diagnosed With Diffuse Large B-Cell Lymphoma. Int J Clin Exp Med (2015) 8(9):15173-81.

26. Marini O, Spina C, Mimiola E, Cassaro A, Malerba G, Todeschini G, et al. Identification of Granulocytic Myeloid-Derived Suppressor Cells (G-MDSCs) in the Peripheral Blood of Hodgkin and Non-Hodgkin Lymphoma Patients. Oncotarget (2016) 7(19):27676-88. doi: 10.18632/oncotarget.8507

27. Gomez S, Tabernacki T, Kobyra J, Roberts P, Chiappinelli KB. Combining Epigenetic and Immune Therapy to Overcome Cancer Resistance. Semin Cancer Biol (2020) 65:99-113. doi: 10.1016/j.semcancer

28. Stone ML, Chiappinelli KB, Li H, Murphy LM, Travers ME, Topper MJ, et al. Epigenetic Therapy Activates Type I Interferon Signaling in Murine Ovarian Cancer to Reduce Immunosuppression and Tumor Burden. Proc Natl Acad Sci (2017) 114(51):E10981-E90. doi: 10.1073/pnas.1712514114

29. Mikkelsen S, Gillberg L, Lykkesfeldt J, Grønbæk K. The Role of Vitamin C in Epigenetic Cancer Therapy. Free Radic Biol Med (2021) S0891-5849 (21):00169-6. doi: 10.1016/j.freeradbiomed

30. Rabal O, San José-Enériz E, Agirre X, Sánchez-Arias J, de Miguel I, Ordoñez R, et al. In Vivo Design and Synthesis of Novel Epigenetic Inhibitors Targeting Histone Deacetylases, DNA Methyltransferase 1, and Lysine Methyltransferase G9a With Efficacy in Multiple Myeloma. J Med Chem (2021) 64(6):3392-426. doi: 10.1021/acs.jmedchem.0c02255

31. Khan O, La Thangue NB. HDAC Inhibitors in Cancer Biology: Emerging Mechanisms and Clinical Applications. Immunol Cell Biol (2012) 90(1):85-94. doi: 10.1038/icb.2011.100

32. Li M, Zha X, Wang S. The Role of N6-Methyladenosine mRNA in the Tumor Microenvironment. Biochim Biophys Acta Rev Cancer (2021) 1875(2):188522. doi: $10.1016 /$ j.bbcan.2021.188522

33. Christmas BJ, Rafie CI, Hopkins AC, Scott BA, Ma HS, Cruz KA, et al. Entinostat Converts Immune-Resistant Breast and Pancreatic Cancers Into Checkpoint-Responsive Tumors by Reprogramming Tumor-Infiltrating Mdscs. Cancer Immunol Res (2018) 6(12):1561-77. doi: 10.1158/23266066.CIR-18-0070

34. Lu Z, Zou J, Li S, Topper MJ, Tao Y, Zhang H, et al. Epigenetic Therapy Inhibits Metastases by Disrupting Premetastatic Niches. Nature (2020) 579 (7798):284-90. doi: 10.1038/s41586-020-2054-x

35. Xie Z, Ago Y, Okada N, Tachibana M. Valproic Acid Attenuates Immunosuppressive Function of Myeloid-Derived Suppressor Cells. J Pharmacol Sci (2018) 137(4):359-65. doi: 10.1016/j.jphs.2018.06.014

36. Xie Z, Ikegami T, Ago Y, Okada N, Tachibana M. Valproic Acid Attenuates CCR2-Dependent Tumor Infiltration of Monocytic Myeloid-Derived Suppressor Cells, Limiting Tumor Progression. Oncoimmunology (2020) 9 (1):1734268. doi: 10.1080/2162402X.2020.1734268

37. Briere D, Sudhakar N, Woods DM, Hallin J, Engstrom LD, Aranda R, et al. The Class I/IV HDAC Inhibitor Mocetinostat Increases Tumor Antigen Presentation, Decreases Immune Suppressive Cell Types and Augments Checkpoint Inhibitor Therapy. Cancer Immunol Immunotherapy (2017) 67 (3):381-92. doi: 10.1007/s00262-017-2091-y

38. Wang HF, Ning F, Liu ZC, Wu L, Li ZQ, Qi YF, et al. Histone Deacetylase Inhibitors Deplete Myeloid-Derived Suppressor Cells Induced by $4 \mathrm{~T} 1$ Mammary Tumors In Vivo and In Vitro. Cancer Immunol Immunother (2017) 66(3):355-66. doi: 10.1007/s00262-016-1935-1

39. Rosborough BR, Castellaneta A, Natarajan S, Thomson AW, Turnquist HR. Histone Deacetylase Inhibition Facilitates GM-CSF-Mediated Expansion of 
Myeloid-Derived Suppressor Cells In Vitro and In Vivo. J Leukoc Biol (2012) 91(5):701-9. doi: 10.1189/jlb.0311119

40. Kim YD, Park SM, Ha HC, Lee AR, Won H, Cha H, et al. Hdac Inhibitor, Cg745, Enhances the Anti-Cancer Effect of Anti-PD-1 Immune Checkpoint Inhibitor by Modulation of the Immune Microenvironment. J Cancer (2020) 11(14):4059-72. doi: 10.7150/jca.44622

41. Bae J, Hideshima T, Tai YT, Song Y, Richardson P, Raje N, et al. Histone Deacetylase (HDAC) Inhibitor ACY241 Enhances Anti-Tumor Activities of Antigen-Specific Central Memory Cytotoxic T Lymphocytes Against Multiple Myeloma and Solid Tumors. Leukemia (2018) 32(9):1932-47. doi: 10.1038/ s41375-018-0062-8

42. Jayaraman A, Sharma M, Prabhakar B, Holterman M, Jayaraman S. Amelioration of Progressive Autoimmune Encephalomyelitis by Epigenetic Regulation Involves Selective Repression of Mature Neutrophils During the Preclinical Phase. Exp Neurol (2018) 304:14-20. doi: 10.1016/j.expneurol. 2018.02.008

43. Brown J, Roberts W. Evidence That Approximately Eighty Per Cent of the Soluble Proteins From Ehrlich Ascites Cells Are Nalpha-Acetylated. J Biol Chem (1976) 251(4):1009-14.

44. Arnesen T, Van Damme P, Polevoda B, Helsens K, Evjenth R, Colaert N, et al. Proteomics Analyses Reveal the Evolutionary Conservation and Divergence of N-terminal Acetyltransferases From Yeast and Humans. Proc Natl Acad Sci USA (2009) 106(20):8157-62. doi: 10.1073/pnas.0901931106

45. Aksnes H, Van Damme P, Goris M, Starheim K, Marie M, Støve S, et al. An Organellar N $\alpha$-Acetyltransferase, naa60, Acetylates Cytosolic N Termini of Transmembrane Proteins and Maintains Golgi Integrity. Cell Rep (2015) 10 (8):1362-74. doi: 10.1016/j.celrep.2015.01.053

46. Allfrey V, Faulkner R, Mirsky A. Acetylation and Methylation of Histones and Their Possible Role in the Regulation of RNA Synthesis. Proc Natl Acad Sci USA (1964) 51:786-94. doi: 10.1073/pnas.51.5.786

47. Narita T, Weinert BT, Choudhary C. Functions and Mechanisms of nonHistone Protein Acetylation. Nat Rev Mol Cell Biol (2019) 20(3):156-74. doi: 10.1038/s41580-018-0081-3

48. Bahl S, Seto E. Regulation of Histone Deacetylase Activities and Functions by Phosphorylation and Its Physiological Relevance. Cell Mol Life Sci CMLS (2020) 78(2):427-45. doi: 10.1007/s00018-020-03599-4

49. Zhao M, Tao Y, Peng G. The Role of Histone Acetyltransferases and Histone Deacetylases in Photoreceptor Differentiation and Degeneration. Int J Med Sci (2020) 17(10):1307-14. doi: 10.7150/ijms.43140

50. Glozak M, Sengupta N, Zhang X, Seto E. Acetylation and Deacetylation of Non-Histone Proteins. Gene (2005) 363:15-23. doi: 10.1016/j.gene.2005. 09.010

51. Villagra A, Cheng F, Wang HW, Suarez I, Glozak M, Maurin M, et al. The Histone Deacetylase HDAC11 Regulates the Expression of Interleukin 10 and Immune Tolerance. Nat Immunol (2009) 10(1):92-100. doi: 10.1038/ni.1673

52. Minucci S, Pelicci P. Histone Deacetylase Inhibitors and the Promise of Epigenetic (and More) Treatments for Cancer. Nat Rev Cancer (2006) 6 (1):38-51. doi: 10.1038/nrc1779

53. Shen L, Orillion A, Pili R. Histone Deacetylase Inhibitors as Immuno modulators in Cancer Therapeutics. Epigenomics (2016) 8(3):415-28. doi: $10.2217 /$ epi.15.118

54. Wagner J, Hackanson B, Lübbert M, Jung M. Histone Deacetylase (HDAC) Inhibitors in Recent Clinical Trials for Cancer Therapy. Clin Epigenet (2010) 1:117-36. doi: 10.1007/s13148-010-0012-4

55. Subramanian S, Bates S, Wright J, Espinoza-Delgado I, Piekarz R. Clinical Toxicities of Histone Deacetylase Inhibitors. Pharmaceuticals (Basel Switzerland) (2010) 3(9):2751-67. doi: 10.3390/ph3092751

56. Kato Y, Yoshimura K, Shin T, Verheul H, Hammers H, Sanni T, et al. Synergistic In Vivo Antitumor Effect of the Histone Deacetylase Inhibitor MS275 in Combination With Interleukin 2 in a Murine Model of Renal Cell Carcinoma. Clin Cancer Res Off J Am Assoc Cancer Res (2007) 13:4538-46. doi: 10.1158/1078-0432.Ccr-07-0014

57. Shen L, Ciesielski M, Ramakrishnan S, Miles KM, Ellis L, Sotomayor P, et al. Class I Histone Deacetylase Inhibitor Entinostat Suppresses Regulatory T Cells and Enhances Immunotherapies in Renal and Prostate Cancer Models. PloS One (2012) 7(1):e30815. doi: 10.1371/journal.pone.0030815

58. Christiansen A, West A, Banks K, Haynes N, Teng M, Smyth M, et al. Eradication of Solid Tumors Using Histone Deacetylase Inhibitors Combined
With Immune-Stimulating Antibodies. Proc Natl Acad Sci USA (2011) 108 (10):4141-6. doi: 10.1073/pnas.1011037108

59. Sillesen M, Bambakidis T, Dekker SE, Fabricius R, Svenningsen P, Bruhn PJ, et al. Histone Deactylase Gene Expression Profiles Are Associated With Outcomes in Blunt Trauma Patients. J Trauma Acute Care Surg (2016) 80 (1):26-32. doi: 10.1097/TA.0000000000000896

60. Kim H, Bae S. Histone Deacetylase Inhibitors: Molecular Mechanisms of Action and Clinical Trials as Anti-Cancer Drugs. Am J Trans Res (2011) 3 (2):166-79.

61. Freundt JK, Frommeyer G, Spieker T, Wotzel F, Grotthoff JS, Stypmann J, et al. Histone Deacetylase Inhibition by Entinostat for the Prevention of Electrical and Structural Remodeling in Heart Failure. BMC Pharmacol Toxicol (2019) 20(1):16. doi: 10.1186/s40360-019-0294-x

62. Orillion A, Hashimoto A, Damayanti N, Shen L, Adelaiye-Ogala R, Arisa S, et al. Entinostat Neutralizes Myeloid-Derived Suppressor Cells and Enhances the Antitumor Effect of PD-1 Inhibition in Murine Models of Lung and Renal Cell Carcinoma. Clin Cancer Res (2017) 23(17):5187-201. doi: 10.1158/10780432.CCR-17-0741

63. Trapani D, Esposito A, Criscitiello C, Mazzarella L, Locatelli M, Minchella I, et al. Entinostat for the Treatment of Breast Cancer. Expert Opin Invest Drugs (2017) 26(8):965-71. doi: 10.1080/13543784.2017.1353077

64. Ruiz R, Raez L, Rolfo C. Entinostat (SNDX-275) for the Treatment of nonSmall Cell Lung Cancer. Expert Opin Invest Drugs (2015) 24(8):1101-9. doi: $10.1517 / 13543784.2015 .1056779$

65. Tomita Y, Lee M, Lee S, Tomita S, Chumsri S, Cruickshank S, et al. The Interplay of Epigenetic Therapy and Immunity in Locally Recurrent or Metastatic Estrogen Receptor-Positive Breast Cancer: Correlative Analysis of ENCORE 301, A Randomized, Placebo-Controlled Phase II Trial of Exemestane With or Without Entinostat. Oncoimmunology (2016) 5(11): e1219008. doi: 10.1080/2162402x.2016.1219008

66. Hashimoto A, Fukumoto T, Zhang R, Gabrilovich D. Selective Targeting of Different Populations of Myeloid-Derived Suppressor Cells by Histone Deacetylase Inhibitors. Cancer Immunol Immunother (2020) 69(9):1929-36. doi: 10.1007/s00262-020-02588-7

67. Qian BZ, Li J, Zhang H, Kitamura T, Zhang J, Campion LR, et al. CCL2 Recruits Inflammatory Monocytes to Facilitate Breast-Tumour Metastasis. Nature (2011) 475(7355):222-5. doi: 10.1038/nature10138

68. Bonapace L, Coissieux M, Wyckoff J, Mertz K, Varga Z, Junt T, et al. Cessation of CCL2 Inhibition Accelerates Breast Cancer Metastasis by Promoting Angiogenesis. Nature (2014) 515(7525):130-3. doi: 10.1038/ nature 13862

69. Duenas-Gonzalez A, Candelaria M, Perez-Plascencia C, Perez-Cardenas E, de la Cruz-Hernandez E, Herrera L. Valproic Acid as Epigenetic Cancer Drug: Preclinical, Clinical and Transcriptional Effects on Solid Tumors. Cancer Treat Rev (2008) 34(3):206-22. doi: 10.1016/j.ctrv.2007.11.003

70. Göttlicher M, Minucci S, Zhu P, Krämer O, Schimpf A, Giavara S, et al. Valproic Acid Defines a Novel Class of HDAC Inhibitors Inducing Differentiation of Transformed Cells. EMBO J (2001) 20(24):6969-78. doi: 10.1093/emboj/20.24.6969

71. Gurvich N, Tsygankova O, Meinkoth J, Klein P. Histone Deacetylase Is a Target of Valproic Acid-Mediated Cellular Differentiation. Cancer Res (2004) 64(3):1079-86. doi: 10.1158/0008-5472.can-03-0799

72. Adeshakin AO, Yan D, Zhang M, Wang L, Adeshakin FO, Liu W, et al. Blockade of Myeloid-Derived Suppressor Cell Function by Valproic Acid Enhanced anti-PD-L1 Tumor Immunotherapy. Biochem Biophys Res Commun (2020) 522(3):604-11. doi: 10.1016/j.bbrc.2019.11.155

73. Bolden J, Peart M, Johnstone R. Anticancer Activities of Histone Deacetylase Inhibitors. Nat Rev Drug Discovery (2006) 5(9):769-84. doi: $10.1038 / \mathrm{nrd} 2133$

74. Gatti L, Sevko A, De Cesare M, Arrighetti N, Manenti G, Ciusani E, et al. Histone Deacetylase Inhibitor-Temozolomide Co-Treatment Inhibits Melanoma Growth Through Suppression of Chemokine (C-C Motif) Ligand 2-Driven Signals. Oncotarget (2014) 5(12):4516-28. doi: 10.18632/ oncotarget. 2065

75. Kroesen M, Bull C, Gielen PR, Brok IC, Armandari I, Wassink M, et al. AntiGD2 $\mathrm{mAb}$ and Vorinostat Synergize in the Treatment of Neuroblastoma. Oncoimmunology (2016) 5(6):e1164919. doi: 10.1080/2162402X.2016. 1164919 
76. Dispirito J, Shen H. Histone Acetylation At the Single-Cell Level: A Marker of Memory CD8+ T Cell Differentiation and Functionality. J Immunol (Baltimore Md 1950) (2010) 184(9):4631-6. doi: 10.4049/ jimmunol.0903830

77. Tseng W, Tsai M, Chen N, Tarng D. Trichostatin A Alleviates Renal Interstitial Fibrosis Through Modulation of the M2 Macrophage Subpopulation. Int J Mol Sci (2020) 21(17):5966. doi: 10.3390/ijms21175966

78. Hong E, Heo E, Song J, Kwon B, Lee J, Park Y, et al. Trans-Scirpusin A Showed Antitumor Effects Via Autophagy Activation and Apoptosis Induction of Colorectal Cancer Cells. Oncotarget (2017) 8(25):41401-11. doi: 10.18632/ oncotarget.17388
Conflict of Interest: The authors declare that the research was conducted in the absence of any commercial or financial relationships that could be construed as a potential conflict of interest.

Copyright (C) 2021 Cui, Cai, Wang and Wang. This is an open-access article distributed under the terms of the Creative Commons Attribution License (CC BY). The use, distribution or reproduction in other forums is permitted, provided the original author(s) and the copyright owner(s) are credited and that the original publication in this journal is cited, in accordance with accepted academic practice. No use, distribution or reproduction is permitted which does not comply with these terms. 